pur ou rnélangé à des matières terreuses abon de dans certaines régions où il forme parfois des filons d'une grande pu ssance; en réservanl à la fabrication des objets de choix les variéiés les plus pures et en utilisant pour la production des verres colorés les varjétés communes, il nộs semble qu'on réaliserail là un grand progrès dans l'induslrie déjà si perfectionnée du verre.

L'idée n'est du reste pas nouvelle, cal plusieurs auteurs ont déjà mentionné la fusion facile clu quartz dans le four électrique. Fn 1893, Moissan a étudié altentivement les propriétés et la votilisalion de la silice dans le four qu'il avait inventé un an auparavant: en plaçant des fragments de cristal de roche dans un creuset de charbon soumis à l'action d'un arc de 350 ampères sous 70 volt.s, il remarqua que la silice entrait en fusion et ne tardait pas à se volaliliser. Les expériences de Gabreau ont de mềme montré que le quarlz fondait facilement dans un creuset chiuffé extérieurement par une série d'arcs disposés circulai. rément.

Bronn lui-même, en vue r'étudier cette fusion, se servit comme matière première de sable du Rhin, dit "sable cristallisè », qui donnait comme produit fondu une suhstance blanchâtre, à arêtes transparentes, el donl l'opacité ne semblail due qu'à l'inclusion d'un grand nombre de petiles bulles gazeuzes dans la masse liquide. Le quarlz ainsi préparé possède l'excellente qualitê de se laisser travailler et tirer facilement en fils longs et fins, lorsqu'il iest à une température suffisamment élevée. Un de ses plus grands avantages est son insensibilité aux changements brusques de température: en outre de cela, le quartz possède une dureté très supérieure à celle du verre ordinaire el même à celie du cristal et il peut, par suite, être utilisé dans un grand nombre de cas où le verre serait un produit bien imparfait.

Comme on peut s'en rendre comple, l'avenir nous réserve bien des chances de réussite dans la fabrication électro-thermique d'un produil, qui, soit de même nalure que le verre, soil ayant toutes ses qualités et présentant sur lui les avantages précilés, viendrait rendre à l'industriel de granils services en réduisant à une valeur ipresque négligeable la main d'œuvre et les frais d'extraction de la matière première.

Une solution très avantageuse, et qui mérite également d'être mentionnée, serait peut-être aussi celle qui a élé proposée récemment par M.Côle et qui est la suivante : utilısation de l'oxygène de lair par la liquéfaction de ce dernier gaz (procédés G. Claude) avec transformation partielle des fours actuels, el installation hydroélectrique comprenanl, par exemple, une série de fours à carbure de calcium et appareils à lifuifier l'air pour la production de la flamme oxyacélylénique, devenue, par ce procédé, économıque et pratique.

Nous laissons aux industriels actifs et compélents le soin de juger par eux-mêmes quel sera le meilleur parti à prendre à la suite de cette élude en vue d'une fahricalion larce et méthorique du verre, espérant que les discussions qu'elle pourra soulever amèneront une solution eflicace de ce problème.

Jean ESCARD, Ingénieur Civil,

Ancien Elève du Laboraloire Cenlral de la Sociéli Internationale des Electriciens.

\section{Traction électrigue des Chemins de fer Suisses}

\author{
Suite du Rapport de la Commission Suisse d'Eludes pour la \\ traction électrique des Chemins de fer \\ Par le Secrétaire général, I'rofesseur Dr Wrssuing (*)
}

Les calculs précédents montrent que, sur les 1200000 chevaux-heures quil sont nécessaires pour le traffc d'un jour d'élé, un sixième au plus, soit 200000 chevaux-heures environ, serait rélcupérable et cela dans le cas d'un système de traction parfail sous ce rapport. Cela r'eprésenterait une économie sensible. Mais il y a lieu de remarquer que cette récupération n'aurait pas partout la même importance. Sur la ligne de Brünig, sur los chemins de fer rhétiens, sur le

(') D’après je Bullelin technique de la Suisse Romando. Pour la premièro partie de cetle étude, voir le no de dócembre de La Houille Blonche.
Gothard, l'énergie ainsi disponible, théoriquement, se monte à un tier's du lravail tolal, tandis que, sur l'ensemble des chemins de fer fédéraux, la proportion est de un huitième, et tombe: à un tr eizième dans le III ${ }^{\circ}$ arrondissement. Si. l'on tient compte, $\in \mathrm{n}$ outrie, du fait que la récupération amène toujours des complications et qu'elle ne peut jamais donner des résultats complets, on reconnait que le souci de la récupération ne doit influencer que secondairement, ou seulement pour certaines lignes, la question du systeme de traction à appliquer ; le bénéfice à réaliser de ce fait sera en tous cas relativement minime

La diminution de dépense en hiver est importanter. Comme on l'a vu plus haut, l'énex"gie nécessaire aux chemins de fer a été calculée non seulement pour un jour d'été, mais aussi pour une journée d'hiver. Le résultat est beaucoup pilus faible dans ce dernier cas ; il y a lieu, par contre, d'ajouter la dépense d'éclairagé et de chauffage.

Comme on le voits le travail de tuaction proprement dit ne s'élève en hiver qu'aux trois quarts environ du tolal de l'été; ; ce rapport tombe pour quelques lignes à 50 pour 100 (sur le Brünig à 25 pour 100) ; par contre, il s'élève plusieurs fois à 90 pour 100 .

Si l'on ajoute à cela le chauffage et l'éclairage, qui montent on moyenne à 13 pour 100 de la dépense de traction, on constate que la dépense d'hiver est, en moryenne, les 77 pour" 100 de celle de. l'été. Remarquons que cette valeur n'est pas un minimum, car on a choisi un jour ouvrable (le minimum se produit le dimanche, le scrvice des marchandises étant supprimé ce'jour-là).

Nous supposerons désormais que les forces hydrauliques disponibles sont munies de réserves d'eau surfisantes pour parcr aux différences d'énergie qui se présentent en hiver et en élé. La constitulion de ces réserves s'imposera d'ellemême pour obvier aux variations de puissance qui serront nécessajres, ainsi que pour compenser" la variabilité des cours d'eau dans les différentes saisons.

Du moment que ces réserves sonl supposées existor, il n'y a plus à tenir compte que de la moyenne annuclle de l'énergie journalière nécessaire, plus brièvement, de l'énergie annuelle. Voici de quelle facon celto quantité a été déterminíe. On s'est basé sur" le trafic réel expriméi en tonneskilomètres par an, obtenu en prenanl la moyenne entro lo trafic d'un jour ouvrable d'été et d'un jour ouvrable d'liver, et en déduisant 7 pour 100 pour la suppression du service des marchandises le dimanche (le trafic des marchandises étant estimé à 50 pour 100 (lu lrafic tolal). Liénergie dissipée par" le chauffage ol l'éclairage a élé comptée pour un tiers de sa valour rélle d'un jour d'hiver, ce qui corresflond à admettre par an 20 jours complets de chauffage ol d'éclairage.

Le travaid moyen de traction, exprimé en tonnes-kilomèIres, représente, pour les voies normales, le 78 pon ${ }^{*} 100$; pour les voies élroites, le 68 pour 100 du travail d'un jour d'été.

Le travail iournalier moyen exprimé en chevaux-houres s'élève à 80 pour 100 de celui d'un jour d'élé ct à 103 pour 100 de celui d'un jour" d'hiver.

L'énergie moyonne nécessaire par jour, pour la traclion, lo chauffage et l'éclairag*e, a cté trouvée égalo à 966000 chovaux, ce quil représente, en calculant par excès, 2400000 chovaux-heures à fournir par les turbines des stations centrales, ce qui correspond à une puissance permanente de 100000 chevaux aux lurbines.

LA PUISSANCE NÉCESSAIRE, ET FN PARTJCULIER SON MAXIMUM. - La délerminalion de la puissance exigée par les divers réseaux et troncons n'est pas utile seulement pour l'étudo iles centrales hydrauliques, mais encore pour celle des canalisations électriques. Nous ne nous occuperons ici, pour le moment, que des forces hydrauliques.

Voici de quelle façon il a été procédé :

Un barême des vitesses d'après les rampes fut établi; cor: 
respondant à peu près à ce qui preut êtue atteint actuellement par la traction à vapeur dans des circonstances favorables. D'après cers vitesses et les valeurs admises plus haut pour la résistanee au roulementy on construisit alors le diagramme des puissances pour un train de 100 tonnes, c'est-à-djre un graphifye donnant, pour chaque calégorie detrains, la purissance en fonction de la rampe.

D'après ce premier diagramme, on put établir, pour chaque trongon de ligne et charue catégorie de trains, un diagramme des trains potr un train de 100 tonnes, en prenant le temps pour abscisse; la puissance en chevaux fut portée en ordonnées sur l'horaire graphique, et cela dans l'hypothèse que le train max"chait sur chaque trongon à la vitesse admise piour la rampe maxima de ee tronçon. Cette hypothèse simptifleatrice peut évidemment être adoptée pour le calcul de la puissance maximum, puisque celle-ci est réellement nécessaire à certains møments ; il est bien entendu, par contre, que la puissance moyenne que l'on obtiendrait en planimétrant ce diagramme des trains serait beaucoup plus élevée que dans la réalitél des ehoses.:

En portant sur l'horaire graphique les diagrammes de tous les trains circulant dans la journée sur la ligne considérée, on oblient finalement le diagramme de chaque troncon poup un train de 100 tonnes.

C.e travail fut fait sur I'horaire d'été 1904 en tenant compte de bous les train périodiques or facultatifis. En prenant enr suile, sur les tableaux de charges des trains des chemins de fer fédérax, les pouds maximum (d'août, pour les trains de voyageur's ; doctobr"e, pour les trains de marchandisesin) de chaque numéro de trains, on construisit un diagrame des puissances pour chaque tronçon, en additionnant toutes les 10 minutes (et parfois à de plus courts intervalles. les puissances exigées par tous les trains en circulation. Pour les lignes autres que celles des chemins de fer fédéraux, on se basa sur les poids maximum indiqués par les horaires de service.

On almit aussi que les trains se suivaient à la distance de blaquage. Pour toules les descentes jusqu'à 6 pour 1000 , øn calcula avec la puissance nécessaire en palier" ; sur les pentes. Les plus forles, on admit que le morvement, se faísait sams apporl d'émergie.

On n'a pas tenu compte spécialement de la puissance nécessaire à l'accélénalion; en effet, avee les accélérations actuelles de $\theta, 1$ à $00,15 \mathrm{~m}$. par sec. sec, on peut considèrer que cethe puissance est targement converte par la supposition: que ehrargue train, dès son départ, exige la puissance nécessaire pour la rampe maxima da trono̧on eonsidéré.

En résumé, toutes les hypothèses faites doivent donner des puissatmees maximum plutôt supérieures à celles qui seront nécessaires en réalité.

On pat ensuile procéder à la confection des diagrammes de puissance de réseaux quelconques, tout simplement en additionnant les ordonnées du même instant. Il en résulta naturellement que, plus les réseaux étaient étendus, plus les courbes de puissances devenaient régulièr's.

Pour pouvoir les nitiliser dans la suite pour le calcul des réseaux de distribution électriques et Galui des machines des stations icentrales, il fut nécessaire de dessiner lés diagrammes de puissances de tous les tronģons. En effeh dans l'exécution futare de Ja traction électrique et d'après la distribution géographitque des forces hydrauliques disponibles, il conviendra diattribuer un point d'alimentation à chaque groupe de tronçons, et à chaque centrale le service d'un certain nombre de ces points d'alimentation.

L'ersendole cu réseau suisse fut partagé en un certain nombre de réseaux ( 140 environ), pour chacu:n desquels on établit le diagramarre des purssances, se basant sur le travaitl journalier total el par consécuent sur la puissance majenue nécessacre 24 heures par iont.

Le rapport entre la puissance maximum thetie puissance moyenne varie dans la plupart trongons entre 7 et 12 ; pout an assez grand nombre, il s'elève à 15 -au 20 (au maxi- mum 37). Par contre les cas où ce rapport tombe jusqu'à 6 sont assez nombreax. et sur quelques lignes isolees, sur le: Gothara notamment, il descend jusqu'à 4 et $3 ; 2$ pour des. tronçons en rampe relativement longs.

On reconnait par ces quelques données que les fluctuations de la prissance exigée sont trè̀s considílables.

En groupant en un petit réseau un ensemble de troncons partant d'un des futur's points d'alimentation, on recornnast. que les fluctuations n'y sont guère plus faibles. Prenons, par exemple, le réseau d'Etzwylen, composé de cinq sections. faisant en tout $161 \mathrm{~km}, 34$. Le diagramme montre, pendant les 22 heures que dure le service journalier, une puissance moyenne :(rapportée à 24 heures) de 1517 . chevaux et présente vingt-cinq maxima et minima, tandis que la puissance maxima đu même réseau s'ẻlève à 10500 chevaux (ce qui représente 6,9 fois la puissance moyemne). La plus forte fluctuation de puissance représente environ le 60 p. 100 de la puissance maxima. Les tronçons dont se compose le réseau, pris un à $u_{n}$, ont des maxima qui se montent au décuple de leur puissanco moryenne; la somme de ces maxima s'élèverait à 14.050 chevaux. Les maxima des diverses sections. ne se produisanli pas simultanément; la réuniom en un seul réseau abaisse le maximum de 14050 et 10500 chevaux, ce qui représente une amélioration qui se chiffre par le rapport. de 10 ì 6,9 .

En ajoutant au rěseau d'Etzwylen les résieaux de. Wir (187, $9 \mathrm{kms})$ et de Rorshhach $(89,3 \mathrm{kms})$, on obtient un réseau de $439 \mathrm{~km}$ de lignes, qui correspond à peu près au $4_{1}^{\text {e }}$ arrondissement des chemins de fer fédéraux, diminué de la ligne Altstâtten-Goire, mais augmenté par contre des lignes secondaires de la région. Ee rapport de. la puissance maxima à la puissance moryenne (toujours comptée sur 24 heures) tombe alors à $\check{b}$, et la plus forte variation ne s'élève plus qu'à 35 pour 100 de la puissance maxima fabstraction faite de la brusque variation qui se produit à I'ouverture et à la clôturé de l'explofitation journalière).

Des constatations analogues peuvent être faites sur tous les réseaux du mềme développiement (soit d'environ 30000 chevaux de puissance maxima aux routes mourices).

Il ne sera pas souvent possible de composer des réseaux plus étendus dẹpendant d'une seule usine génératrice ; l'exammen des travaux déjà faits permet de conclure qu'il n'est guère possible de faire descendre au-dessous de 5 le rapport entre la puissance maxima et la puissance moyenne ; ce rappert doil done être consixléré comme la limite inférieure de ce qui pourra êlare obtenu dans cet ordre d'idées.

InStALlation DES USINES CENTRALES. - Les résultats que l'on vient de lire, extraits du travail de M. l'ingénieur Thormann pourront servïr de base à l'étude des installations pour la force motrice et la distribution. Nous allons $\in$ n tirer ${ }^{*}$ immédiatement quelques conclustons.

Les usines centrales devront tout d'abord être capables de supporter des variations énormes de débit ; même en groupant les lignes en réseaux étendưs alimentés par dés usines hydrauliques aussi puissantes que possible, les machines et l'ensemble des installations devront pouvoir fournir à l'oceasion le quintuple de leur puissanice moyenne. Pour les réseaux de faible développement, dont. les centrales seront relativement petites, et pour les stations de transformation, il arrivera facilement que les puissance maxima s'élèveront cu décuple de la puissance moyenne.

on se mend compte à 'la réflexion qu'il m'y a pas possibilite, dans l'êtat actuel des choses (êt peut-être pour toujours), de songer à égaliser les puissanices demandés aux centrales par 1'empioi de batteries d'accumulateurs. On est donc amené à imposer aux asimes génératrices et à leurs réserves hydrauliques le soin de parer aux variations de puissance ; ce: fait limite te choix des empilacements d'usines à ceux qui sont situés près des lacs, car on ne peut songer, au point de vue èconomique, à faire des installations où on laisserait. s'écouler, sans l'utíliser, l'eau surabondante aux instants 
de charge moyenne. Ce dernier gerre id'usines ne peut donc être employé qu'en connéxion avec des installations possesdant des réserves hydrauliques. Comme, d'autre parth l'installation de réserves ne peut se faire ércnomiquement qu'avec de haules chutes, ce sunt ces deraières yu'll conviendra surtout d'utiliser.

Dans tous lès cas où des réservoirs ne pourront pas êtr"e placés à proximité des centrales, il laudra chercher à créer des réstrves indirectes, au moyen de moteurs électriques, de pompies ou de réservoirs à haute chute placés à une certaine distance.

Nouls allons essayer de nous rendre compte, superficiellement au moins, de la puissance totale des chutes nécessaires. Nous pourrons admettee en première approximation que le rendement est à pell près le même à la plus forte charge qu'à la puissance moryenne, c'est-à-dire que ce rendement est égal à celui qui a été supposé plus haut dans le calcul de l'énergie. En effet, le rendement des moteurs, du réglage et des transformateurs est plus élevé à forte charge qu'à puissance moyenne, tandis que pour des canalisations élactriques, c'est le contraire.

Ici encore, nous poutrons adopter 45 pour 100 comme rendement de la transmission des turbines aux rails, même dans les cas d'un. système très désavantagreux, d'autant plus que nous pouvons admettre que la présence d'accumulateurs électriques n'amènerait pas de changements notables dans ce rapport ; mais nous ne calculerons ici qu'avec 40 pour 100, camme dans la première partie de ce rapport ; en outre, nous adopterons encore les rapports de cinq entre les puissances maxima et moyenne.

Les 100000 chevaux de puissance moyenne donneront alors 500000 chevaux comme puissance des turbines des stations centrales; ces 500000 chevaux seraient employés en plein 4 heures 45 minutes par jour, en moyenne $\left(\frac{24}{5}=4^{\mathrm{h}} 45\right)$ Ceci dans l'hypathèse de très grandes usines génératrices ; s'il devient nécessaire de fractionner ces usines, il faudra multiplier les 100000 chevaux pax un nombre plus grand que 5.

Si Ia récupiération sur les pentes grrive à pouvoir se faire pratiquement, l'utitisation de la puissance maxima diminuera ; mais cette puissance maxima elle-même ne diminuera pas beaucoup ; ce ne sera guère que sur l'énergie jourtnaibere ou annelie qu'il y aura bénéfice.

\section{A propos de la meilleure utilisation des Chutes}

Les forces hydrauliques ont acquis, aujourd'hui, une valeur incomparablement plus grande que celle qu'elles avaient it y a quelques années, depuis qu'il est possible, grâce au développement du. transport de l'énergie électrique, de transmettre à de grandes distances et avec succès des quantités considérables d'énergie. On peut ainsi employer de nombreuses: forces hydrauliques éloignées, auxquelles il n'était pas permis de songer autrefois et, en considération de la hausse des prix de la houille, il est hors de doute que l'emploi de ces forces hydrauliques ne se propage rapidement.

Deux inconvénients, cependant, viennent amoindrir la valeur de ces forces hydrauliques. C'est, d'abord, l'irrégulamité de la putissance qui, parfois, après des pluies abondantes, amène une surabondance d'eau dont l'utilité est nutle, tandis qu'à d'autres moments, surtout pendant les grands froids, ou les temps de sëcheresse, le débit courant devient très faible et, par suite, insuffisant pour procurer l'effort nécessaire au fonctionnement complet des installations. On peut remédier avantageusement à cet inconvé-

nient en aménageant des barrages, surtout dans les pays de montagnes, afin d'y accumuler les eaux de pluie de toute l'année, et de les distribuer chaque jour suivant les besoins.

Le second inconvénient, dont l'aplanissement fait l'objet KW.

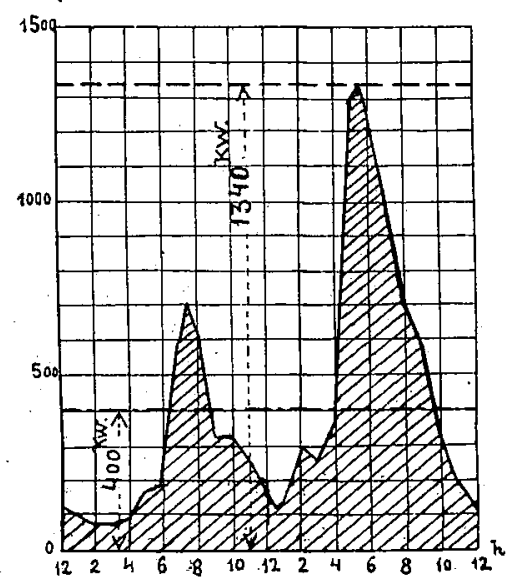
de l'exposé ci-après, consiste en ce que les forces hydrauliques disponibles sont très souvent incomplètement utilisées par les consommateurs, de sorte qu'une grande partic de l'eau s'écoule du barrage sans rendre de services, et que le facteur de charge (c'est-à-dire le rapport existant entre l'énergie effectivement utilisée et celle réalisable par la force hydraulique dont on dispose) est très minime. La charge la plus désirable serait celle qui donnerait un rende. ment stable pendant 24 heures, c'est-ì-dire un rendement dont chaque kilowatt produit par l'énergie hy-

FIG. 1. - Diagramme d'une central d'éclairage (Décembré)

Maximun $=1340 \mathrm{kw}$

Moyenne de 24 heures $=400 \mathrm{kw}$

$\frac{\text { Maximum }}{\text { Moyenne }}=3,35$.

Facteur de charge $=30 \%$ draulique serait complètement utilisé par le consommateur. Dans certains genres d'industrie, le travail est bien de 24 heures à pleine charge, comme, par exemple, dans les fabriques électro-chiniques; dans les moulins, dans les papeteries, etca; au contraire, dans la grande majorité des usines, le travail est très variable ou intermittent. Ce cas concerne surtout les centrales d'éclairage électrique, de distribution de force, et les centrales pour la traction électri-

kW

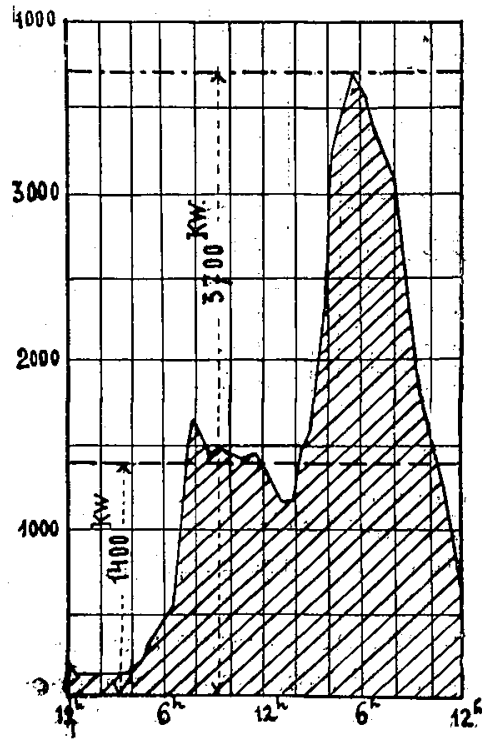

Fig. 2. - Diagramme d'unecentrale pour éclairage, foree et traction.

Maxïmum $=3700 \cdot \mathrm{kw}$.

Moyenne de 24 heures $=\mathrm{r} 400 \mathrm{kw}$ $\frac{\text { Maximum }}{\text { Moyenne }}=2,64$.

Facteur de charge $=38 \%$. nué, ear le rendement d'une station hydraulique dépend, en première ligne, d'un facteur de charge élevé. Si ce dernier est faible, la concurrence de la vapeur ou des moteurs calorifiques est plus à craindre et, lorsque cette que, qui n'ont, la plupart du temps, qu'un facteurde charge très faible (souvent seulement de 30 ou 50 pour cent). Les figures I et 2 représentent un graphique de centrale qui fonctionne effectivement pour l'éclairage et pour actionner de petits moteurs. et qui n'a qu'un facteur de charge de 30 à 38 pour roo. La figure 3 représente un graphique de chemin de fer (chemin de fer de la Valteline) n'ayant que 28 pour roo de facteur de charge. La figure 4, enfin, donne le graphique d"une fabrique travaillant dix heures, et où l'eau reste inutilisée pendant 14 heures par jour, ce qui représente seulement une utilisation de 42 \% de la charge.

Dans de semblables stations hydrauliques, l'utilisation est donc très minime, et le ren. dement en est fortement dimj- 